\section{Questión}

Periodismo / Comunicación ISSN 1669-6581
- Av. $44 \mathrm{~N}^{\circ} 676,1^{\circ}$ piso

CP 1900 - La Plata - Argentina

www.perio.unlp.edu.ar/question

Desafíos educativos en tiempos de pandemia

Lic. Victoria Gagliardi

DOI: https://doi.org/10.24215/16696581e312

\title{
Desafíos educativos en tiempos de pandemia
}

\section{Educational challenges in times of pandemic}

\author{
Lic. Victoria Gagliardi / victoriagagliardi@gmail.com \\ Licenciada y Profesora en Comunicación Social (UNLP). Docente e investigadora UNAJ.
}

La cuarentena lo cambió todo. Desde que la Organización Mundial de la Salud declaró la pandemia de COVID-19, el confinamiento total en Argentina nos enfrentó a un escenario inédito en el que fue necesario reconfigurar todos los aspectos de la vida cotidiana. La suspensión de clases, efectiva desde el 16 de marzo en nuestro país, llevó a todas las instituciones educativas a reformular sus propuestas existentes y promovió la virtualización compulsiva de las propuestas áulicas. En este contexto, por primera vez en la historia todos los niveles se vieron obligados a adaptar su propuesta hacia el ciberespacio.

Este proceso tuvo adeptos y detractores. Mientras la inmensa mayoría de los actores educativos dispusieron una rápida puesta en marcha que incluyó capacitaciones docentes y la adaptación de las currículas a entornos de enseñanza virtual, otros optaron por la suspensión hasta junio. También existieron resistencias entre quienes tomaron el guante de la educación a distancia, que llevaron a impulsar algunas de las propuestas más interesantes: convenios con compañías de telefonía celular para garantizar el acceso libre a portales educativos, creación de sitios de enseñanza específicos para el trabajo en esta coyuntura, adaptación de las cursadas hacia modalidades de aula virtual con propuestas sincrónicas y asincrónicas. 
Un abanico de políticas públicas que rápidamente se articularon con las instituciones educativas (una de las maquinarias institucionales más diversas y complejas del país) para aceitar la rueda y permitirle que siga girando, aún cuando todo lo demás se había detenido.

Hay que destacar que las clases continúan gracias a todos los esfuerzos institucionales que se han realizado para sostenerla y esto constituye una primera victoria para quienes entendemos la educación como un derecho humano. Esta educación pandémica, caracterizada como exclusivamente a distancia y con modalidades diversas de abordaje pedagógico, ofreció una forma de paliar la crisis educativa en un contexto en el que dar clases presenciales se había convertido en un atentado a la salud pública. Sin embargo, esto no garantizó el éxito sino que abrió la puerta a otros interrogantes.

Las clases desde casa, sumadas a los problemas que acarrea la cuarentena total, implicaron un estrés para todos los actores del sistema educativo. Muchos de los esfuerzos estatales e institucionales en estas primeras semanas estuvieron dedicados a canalizar esas angustias, habilitando espacios de consulta y diálogo. Otras propuestas estuvieron orientadas al desarrollo de habilidades y competencias que, en todos los sentidos, debían adoptarse con urgencia para constituir una experiencia inédita que probablemente se convierta en objeto de estudio de las ciencias sociales en los próximos años. Con el foco puesto en el presente, las acciones se concentraron en aplacar la urgencia con urgencia para contener el sistema educativo en todos sus niveles. Tic, tac.

Educar a distancia, viejos conocidos

Las primeras experiencias de educación a distancia son anteriores a la digitalización de la información producida a partir de la aparición de los ordenadores personales e internet. Frente al paradigma hegemónico de la escuela, la tiza y el pizarrón, numerosas experiencias a lo largo del siglo XX dejaron un sedimento fértil para pensar una educación que innove en los tiempos y espacios de la clase tradicional.

Algunas reconocidas prácticas en la región utilizaron a medios de comunicación como los medios gráficos (Freire, 1973) o las radios comunitarias (Kaplún, 1984) para proyectar métodos de enseñanza por fuera de los muros del aula. Posteriormente en Argentina, a partir de la década de 1980 las experiencias de educación a distancia ampliaron sus horizontes en los inicios de la revolución digital. Mientras se abrieron las posibilidades para consumir y compartir 
información, el acceso a internet se fue extendiendo en el mundo y las instituciones educativas comenzaron a participar en esas redes de conocimiento.

Con el tiempo, la ampliación en el acceso a redes e internet habilitó una verdadera revolución en los modos de acceso al conocimiento a partir de iniciativas institucionales, que conformaron las primeras experiencias de educación virtual. Las condiciones materiales para el acceso al saber se habían transformado antes de la pandemia frente a la posibilidad de acceder a múltiples espacios, entendidos como entornos donde existe interacción y en los que el conocimiento circula (Burbules, 2014).

Estas referencias nos permiten realizar un recorrido arqueológico para reconstruir cómo llegamos hasta aquí pues, lejos de lo que a veces se piensa, la educación a distancia no es nueva, por lo tanto no sería correcto afirmar que estamos empezando de cero. Pero también es cierto que nadie estaba preparado para el volumen y la complejidad que implicaría virtualizar todos los niveles de enseñanza de Argentina en un contexto de pandemia, con una población duramente golpeada por la crisis económica y múltiples brechas de acceso al mundo digital aún sin resolver.

Estamos en cuarentena, ¿ahora qué?

Mientras intentamos resolver la transformación de nuestras currículas, adaptamos nuestras propuestas para cumplir con el objetivo de continuar con la enseñanza sin presencia física. En este contexto, es importante que seamos claros: las clases virtuales no sustituyen la presencialidad, son otra cosa. Por lo tanto, todas las planificaciones previas a la cuarentena deben ser reestructuradas, repensadas y aplicadas teniendo en cuenta el contexto de pandemia.

Existen muchas formas de adaptar una propuesta hacia un modelo asincrónico o sincrónico. No depende del área de trabajo o disciplina, sino más bien de la evaluación del tipo de alfabetización digital de los sujetos educativos, de sus habilidades técnicas y la capacidad que tengan para resolver los problemas que puedan surgir en este proceso. Pensemos un ejemplo: mientras algunas propuestas se han adaptado a la realización de actividades bajo algún formato de campus, blogs o plataformas digitales, en otros casos las iniciativas se volcaron al diseño de una serie de videoconferencias, cuya ventaja se basa en la posibilidad de dialogar en vivo con los estudiantes. 
En una población con dificultades de acceso a internet o con pocos dispositivos personales para conectarse a la web la opción de trabajar de forma sincrónica puede ser un problema. Asistir a una videoconferencia implica destinar un dispositivo con conexión a internet de forma exclusiva a una actividad durante un período determinado de tiempo. ¿Todos nuestros estudiantes pueden cumplir este requisito? ¿qué pasa si alguien más en el hogar necesita usar ese dispositivo para trabajar? ¿la calidad de su conexión les permite aprovechar la dinámica sin desconectarse? ¿qué tan amigable es esta propuesta si nos conectamos utilizando datos móviles? ¿estamos pensando la videoconferencia como una clase que dialoga con los estudiantes presentes, o más bien es una conferencia transmitida por internet?

Si no fuera así, la opción por modalidades asincrónicas nos encuentra frente a la dificultad que supone trabajar con una herramienta que puede resultar desconocida y a partir de la cual es necesario incorporar ciertas habilidades con el objetivo de poder utilizarla de forma autónoma. ¿Son nuestras interfaces lo suficientemente intuitivas como para garantizar que estudiantes y docentes sin experiencia puedan cumplir con los objetivos de la clase?

\section{Es momento para experimentar}

Nos enfrentamos a dilemas complejos cuando debemos adaptar nuestras propuestas a contextos excepcionales como éste. Seguramente podremos obrar mejor si contamos con información sobre las características de los estudiantes y sus docentes. Por este motivo, no hay una sola manera de resolver los problemas que surgen frente a una virtualidad compulsiva, urgente y que garantice la inclusión (porque si hay algo que debe preocuparnos como educadores, es enseñar con todos nuestros estudiantes adentro -del campus, de la videollamada o de la herramienta que sea-).

Este desafío supone reconocer las otras corporeidades que se configuran desde lo virtual. Encontrar nuevos modos de habitar la clase, incluso desde la virtualidad. Aportar humanidad al entorno web, que a veces puede resultar poco amable para algunos. Hay que dotar a las clases de sentido: ¿para qué usar esta plataforma? ¿para qué esta actividad? ¿Cuáles son los objetivos de esta tarea? ¿Qué criterios de corrección se aplicarán? ¿Pueden ser los mismos parámetros que utilizamos en la clase presencial o habrá que pensar nuevas formas de evaluar en este contexto? 
La práctica se puede planificar en tiempo presente escuchando a los estudiantes, alterando espacios y tiempos de clase, interactuando con ellos y debatiendo la actualidad. El potencial de las historias como motor cultural se resignifica en contextos donde el aprendizaje se puede transformar en una experiencia narrativa.

Aquello que usualmente tenía lugar en el aula, ahora tiene que ser explicitado en los espacios de interacción y repensado para estos entornos. Es importante que trabajemos en una impronta propia para elaborar debates con eje soberano, fortaleciendo la participación y la inteligencia colectiva. En este contexto de profunda angustia generalizada, no alcanza con brindar a nuestros estudiantes la última actualización con las consignas de trabajo. Se requiere de diálogos, intercambio, participación, salir de la lógica broadcasting para que una propuesta educativa tenga sentido en un entorno donde la colaboración es un rasgo característico de las culturas digitales.

El contexto de pandemia puede abrir la posibilidad para la innovación pedagógica, permitiéndonos repensar las planificaciones hacia nuevas experiencias. En un momento incierto, es tiempo de imaginar de qué manera podemos apropiarnos de estos entornos y salir del terreno de la táctica para pasar al de la estrategia a través de la colaboración colectiva. Son tiempos difíciles, críticos, estamos en cuarentena y aislados. En este contexto, la mejor resistencia posible es unirnos y el trabajo en equipo es fundamental: este es el momento de codiseñar. Así como nadie se salva solo, nadie encuentra en soledad la solución ya que la docencia también es una construcción colectiva, afectiva y emancipadora. Vale la pena aprovechar esta oportunidad para construir nuevas utopías.

Bibliografía

APARICI Roberto, 2011, "Principios pedagógicos y comunicacionales de la web 2.0". Revista Digital LaEduc@ción, Washington, №145.

ALBARELLO Francisco y MIHAL Ivana, 2018, "From canon to school fandom: \#Orson80 as transmedia educational storytelling", Comunicación y Sociedad Guadalajara, № 33, 223-247.

BUCKINGHAM David, 2008 (2007), Más allá de la tecnología. Aprendizaje infantil en la era de la cultura digital, Buenos Aires: Manantial 
BURBULES Nicholas, 2014, "El aprendizaje ubicuo: nuevos contextos, nuevos procesos" Revista Entramados: Educación y Sociedad, Mar del Plata, № 1.

DUSSEL Inés. y QUEVEDO Luis, (2010), Educación y nuevas tecnologías: los desafíos pedagógicos Buenos Aires: Santillana.

FREIRE, Pablo, (1973), ¿Extensión o comunicación? La concientización en el medio rural. México: Siglo XXI

KAPLÚN, Mario, (1978), Comunicación entre grupos: el método del cassette-foro. Bogotá: Centro Internacional de Investigaciones para el Desarrollo, CIID

JENKINS Henry, (2008), Convergence Culture: la cultura de la convergencia de los medios de comunicación. Barcelona: Paidós

MAGGIO, Mariana, (2018), Reinventar la clase en la Universidad. Buenos Aires: Paidós MOLAS CASTELLS Nuria, (2018), La guerra de los mundos. Narrativa Transmedia en educación. Barcelona: UOC.

SCOLARI Carlos, 2016, "Estrategias de aprendizaje informal y competencias mediáticas en la nueva ecología de la comunicación". Revista TELOS (Cuadernos de Comunicación e Innovación), Madrid, № 103, ISSN: 0213-084X pp. 1/9. 